\title{
Multirate Processing of the ECG using Filter Banks
}

\author{
VX Afonso ${ }^{\dagger}$, WJ Tompkins ${ }^{\dagger}$, TQ Nguyen ${ }^{\dagger}, \mathrm{S}$ Luo $^{\#}$ \\ TDepartment of Electrical and Computer Engineering, University of Wisconsin-Madison, WI, USA \\ \#Burdick Inc, , Milton, WI, USA
}

\begin{abstract}
We propose an algorithm which can potentially perform multiple ECG processing tasks using a filter bank $(F B)$. One set of filters in the FB decomposes the ECG into uniform frequency subbands. Since the subbands have a narrower bandwidth than the input ECG, they can be downsampled to get a lower rate subband signal. Time and frequency dependent processing can be performed at a lower rate and hence reduce the computation cost. $A$ beat detection algorithm is presented which has minimal detection latency and good beat detection performance on the MIT/BIH database. The ECG can be enhanced by processing the subbands to remove noise. Features computed from the subbands can be used to distinguish between some ventricular and sinus beats. The FB offers a strategy to perform multiple tasks on the ECG using one set of filters operating at a computationally efficient rate.
\end{abstract}

\section{Introduction}

Many digital signal processing (DSP) solutions for processing the electrocardiogram (ECG) are reported in the literature [1-4]. Some of these processes include beat detection, ECG enhancement, beat classification and noise alert. A crucial task to ECG monitoring and diagnostic systems is ECG beat detection. During a stress test the ECG is acquired from a patient who is performing some form of physical exercise. The ECG acquired during a stress test is usually contaminated with noise. It is important to enhance the stress ECG so that the dynamic changes in the morphology of the ECG induced by the exercise can be studied. ECG interpretive systems which distinguish between various types of arrhythmias are also useful. A classification algorithm which extracts discriminatory features from the ECG is usually part of such interpretive systems. A noise alert feature indicates the fidelity of the ECG.

Existing DSP solutions to the above tasks are accomplished using separate preprocessing filters, each of which is useful for its respective task only. Furthermore the solutions operate at the input rate of the ECG. The strategy presented in this paper seeks to use one set of preprocessing filters to accomplish multiple tasks of the ECG at a rate lower than that of the input ECG.
We propose a filter bank (FB) based system to accomplish multiple tasks on the ECG using one set of filters. The design and usage of FBs are presented in various sources in the literature [5-7]. Figure 1 shows that a FB contains one set of analysis filters which decompose an input signal into various frequency subbands with uniform bandwidth. The subbands have a smaller bandwidth than the input signal and thus can be downsampled. Time and frequency dependent processing can be performed on each subband at the lower rate. Thus the ECG can be processed in a multirate strategy because of the downsampling process.

Section 2 explains that it is possible to design a set of synthesis filters which reconstruct the upsampled subbands to yield a perfect reconstruction of the input signal. Section 3 presents an algorithm which analyzes the subbands to detect beats in the ECG. Section 4 presents an algorithm which processes the subbands to reduce the level of noise in the stress ECG. Section 5 proposes a strategy to compute features from the subbands to distinguish between sinus beats and ventricular beats.

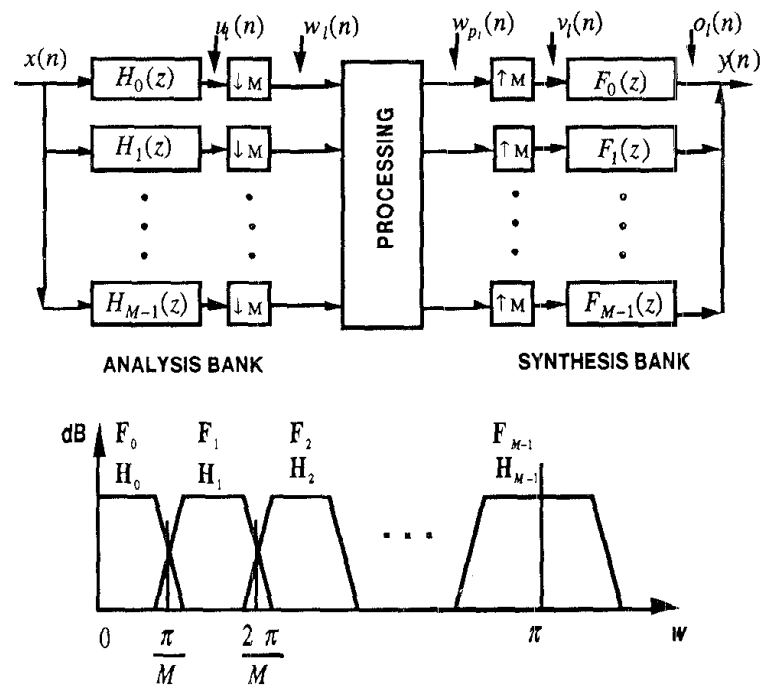

Figure 1. Time and frequency dependent processing can be performed with a multirate strategy because of the downsampling blocks. The ideal magnitude responses of the filters are shown. 


\section{Filter bank review}

Assume that the FB contains $M$ analysis and synthesis filters, each of length $\mathrm{L}$ (see Figure 1 ). The analysis filters $H_{l}(z), l=0,1, \ldots, \mathrm{M}-1$, bandpass the input signal $X(z)$ to produce the subbands $U_{l}(z)$ :

$$
U_{l}(z)=H_{l}(z) X(z) \quad l=0,1, \ldots, M-1
$$

The effective bandwidth of $U_{l}(z)$ is $\frac{\pi}{M}$, and the downsampling process keeps one sample out of every $M$ samples. The downsampled subband signal is

$$
W_{l}(z)=\frac{1}{M} \sum_{k=0}^{M-1} U_{l}\left(z^{\frac{1}{M}} \mathrm{~W}^{k}\right) \quad l=0,1, \ldots, M-1
$$

where $W=e^{-j \frac{2 \pi}{M}}$. The analysis filters can be efficientily operated at $\frac{1}{M}$ the input rate $[5,6]$. The downsampled subbands can be processed according to a specific application to obtain $W_{p_{l}}(z)$. References [5-7] present techniques to design a set of analysis/synthesis filters which can perfectly reconstruct the input signal when no processing takes place, i.e. $W_{l}(z)=W_{p_{l}}(z)$. The upsampled subbands are then given by

$$
V_{l}(z)=\frac{1}{M} \sum_{k=0}^{M-1} H_{l}\left(z \mathrm{~W}^{k}\right) X\left(z \mathrm{~W}^{k}\right) l=0,1, \ldots, M-1
$$

and the output signal is given by

$$
Y(z)=\sum_{k=0}^{M-1} T_{k}(z) X\left(z \mathrm{~W}^{k}\right)
$$

where

$$
T_{k}(z)=\frac{1}{M} \sum_{l=0}^{M-1} H_{l}\left(z \mathrm{~W}^{k}\right) F_{l}(z) \quad k=0,1, \ldots, M-1
$$

Equation (4) shows that the output $Y(z)$ has aliased components of the input. Stress ECG enhancement, as explained in Section 4, requires perfect reconstruction (PR) of the input signal at the output when there is no processing block in Figure 1. PR is achieved when $T_{k}(z)=0$ for $k=1, \ldots, M-1$ and $T_{0}(z)$ is a delay. The relationship between the input and output in Figure $I$ is then given by

$$
y(n)=c x(n-k)
$$

where $k$ is the system delay, and $c$ is a constant gain factor. The output of the $\mathrm{FB}$ is $Y(z)=T_{0}(z) X(z)$ where $T_{o}(z)=c z^{-k}$ is the overall system function. Techniques to design a PR FB are given in [5-7]. Readers may find design programs at the URL address http://saigon.ece.wisc.edu/ waveweb/QMF.html.
Figure 2 shows that the downsampled subbands are input to processing blocks which perform beat detection, beat classification, ECG enhancement, and noise alert functions.

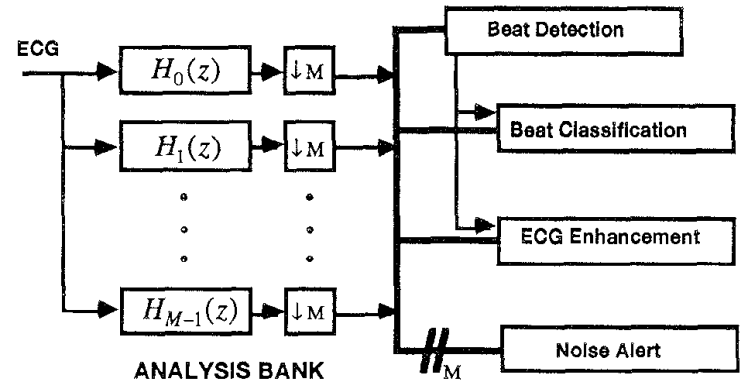

Figure 2. The FB provides one set of filters which can be used to perform multiple ECG processing tasks. The downsampling blocks reduce the number of computations per second.

It is necessary that the analysis filters used for ECG processing have linear phase. The FB can have no phase distortion with respect to the input (i.e., $T_{0}(z)$ has linear phase) even if the analysis and synthesis filters have nonlinear phase responses. However since it is important to have the same delay of the fiducial points (e.g., $R$ wave) across the subbands the analysis filters should have linear phase and the same group delay.

The PR property of the FB is useful for stress ECG enhancement where it is necessary to perform time and frequency dependent processing of the subbands before reconstruction. The PR property ensures that errors due to the nonideal nature of the filters are eliminated.

For the study in this paper a 32-channel FB was designed based on an algorithm from [8]. The analysis and synthesis filters have linear phase responses and have the PR property. ECG records (sampling rate of $360 \mathrm{~Hz}$ ) were acquired from the MIT/BIH database. The $[0,180] \mathrm{Hz}$ frequency bandwidth of the input ECG is thus decomposed into 32 uniform frequency subbands, $\{[0,5.625],[5.625$, $11.25], \ldots,[174.375,180]\}$.

\section{ECG beat detection}

Beat detection algorithms reported in the literature incorporate one bandpass filter which maximizes the output signal-to-noise-ratio of the QRS complex [1]. The bandpass signal is then squared and input to a moving window integrator (MWI) to get an estimate of its energy. The energy peaks around the QRS complex and serves as a feature which indicates the presence of a beat. Adaptive signal and noise levels, and a threshold constitute the logic which determines if a peak in the MWI output is a QRS complex or not.

We designed a beat detection algorithm incorporating similar logic to the above but also using multiple features 
computed from the subbands of the FB [9]. For example one of the features is computed by taking the absolute value of the subbands and then adding across the subbands corresponding to the $[5.625,22.5] \mathrm{Hz}$ range. When input to a MWI filter this feature peaks near the QRS complex. Figure 3 shows a sample ECG segment, combined subbands, MWI output and peaks in the MWI output. Adaptive signal and noise levels and similar logic to that in the traditional algorithm [1] are then used to determine if the peaks are due to the QRS complex or noise.

The algorithm was tested on the MIT/BIH database and had a sensitivity of $99.59 \%$ (374 false negatives) and a positive predictivity of $99.56 \%$ (406 false positives) [9]. The performance is comparable to other results presented in the literature. Furthermore the algorithm is a real-time detection algorithm since it detects beats with a minimal latency of about $266 \mathrm{~ms}$. Since the subbands are downsampled, the FB-based beat detection algorithm only needs to operate at $\frac{1}{M}$ the input ECG rate and this yields a computationally inexpensive beat detection process.

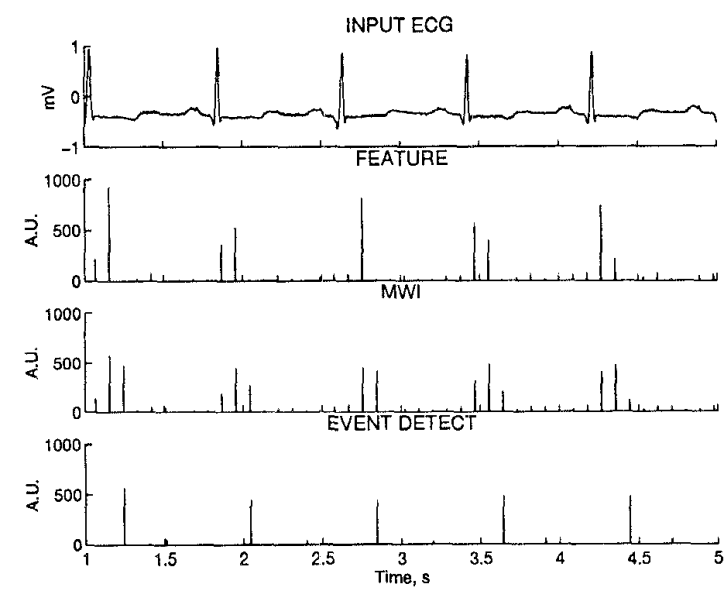

Figure 3. Beat detection can be performed by using adaptive signal and noise thresholds on the MWI output. The detection process is performed at a reduced rate as compared to the input ECG.

\section{ECG enhancement}

The FB system enables processing of a signal in a specific time period and a specific frequency region $[3,4]$. Since the filters of the FB are designed to have the perfect reconstruction property the subband signals can be synthesized to obtain a perfect reconstruction of the input signal. In order to enhance the stress ECG the subband coefficients in Figure 1 can be selectively attenuated based on time and frequency domain information. For example during the QRS region of the ECG the subbands are not attenuated in any of the subbands. But during the non--QRS region the higher frequency subbands are attenuated since the $\mathrm{P}$ and $\mathrm{T}$ waves do not contain high frequencies.

The upsampling operation inserts $M-1$ zero data points in the subband, and this further contributes to computational efficiency during the operation of the synthesis filters. Figure 4 shows a noise-free ECG beat, a stress ECG beat obtained by adding noise acquired during a stress test, and the enhanced beat as obtained by the FB based enhancement algorithm.

To remove low frequency noise a high-pass filter can be incorporated in the lowest subband. A delay should be inserted in the remaining subbands to compensate for the filter delay of the high-pass filter. Since it operates on the downsampled subband, the high-pass filter operates at a lower rate than that of the input ECG.

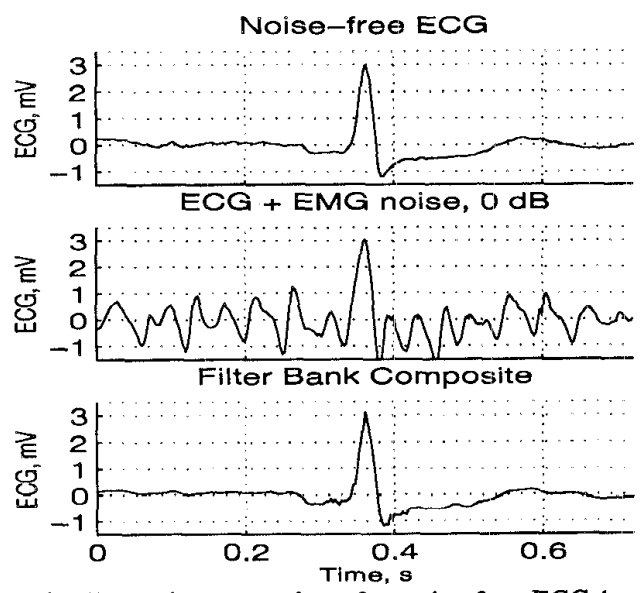

Figure 4. Illustrative examples of a noise-free ECG beat, the same noise-free beat with stress ECG type of noise, and the enhanced beat obtained by using a filter bank. The enhanced beat resembles the underlying noise-free ECG beat.

\section{ECG beat classification}

Algorithms which distinguish between normal sinus beats, ventricular tachycardia or ventricular fibrillation incorporate discriminatory features computed from the ECG. [2] presents an algorithm which extracts parameters from the peaks in the frequency spectrum of an ECG segment. The parameters include the normalized energy in a band around the peak frequency; and normalized energy in bands around harmonics of the peak frequency. Normal sinus beats and ventricular ectopic beats can be distinguished in this manner.

The FB decomposes the ECG into subbands representing the signal in different frequency bands. As we stated previously the subband signals $O_{1}(n)$ can be combined to result in a perfect reconstruction of the input when there is no processing block in Figure 1. We thus 
performed a pilot study in which we combined various sets of subbands $O_{l}(n)$ and computed the energy in a $\pm 50 \mathrm{~ms}$ time window around the $\mathrm{R}$ wave of the QRS complex.

For example subbands 0,1 , and 2, $([0.0,16.8 \mathrm{~Hz}])$ and $0,1,2,3,4([0.0,28]) \mathrm{Hz}$ were combined and the energy in a window around the $R$ wave was computed. The ratio of the former to the later parameter was computed to determine the proportion of the energy in the $[0,16.8] \mathrm{Hz}$ band. This feature can distinguish between normal sinus beats which have a sharp, and narrow QRS complex, and some ventricular beats (e.g. premature ventricular contractions, PVCs) which usually have a rounded, and broad QRS complex.

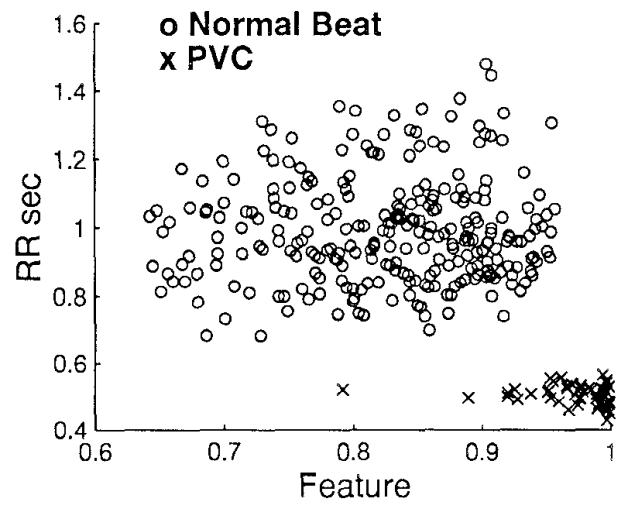

Figure 5. Features extracted from the subbands are useful in distinguishing between some sinus beats and ventricular beats.

Figure 5 plots the above feature versus the RR interval (computed with respect to the previous beat). There is a clear separation of the features from the ventricular and normal sinus beats computed from a record in the MIT/BIH. Normal sinus beats have a sharp QRS complex and thus have higher frequency components than some ventricular beats. However, it is important to note that these features are not useful to distinguish normal beats from all types of ventricular beats. The QRS complexes in certain ventricular ectopic beats can be about $120 \mathrm{~ms}$ which is only slightly more than the $80 \mathrm{~ms}$ (or less) width expected in normal sinus beats. Thus more features similar to these along with a classification algorithm should be designed which is useful in distinguishing between arrhythmias.

\section{Summary}

A multirate filter bank-based strategy which is potentially useful to perform multiple ECG processing tasks such as beat detection, ECG enhancement, beat classification, and noise alert function is proposed. The FB decomposes the input ECG into subbands with uniform frequency bandwidths. A beat detection algorithm is presented which has good beat detection performance on the MIT/BIH database. The ECG can be enhanced by processing the subbands in a time and frequency dependent manner, and then reconstructing. Pilot studies in computing discriminatory features from the subbands seem promising in distinguishing between normal sinus and ventricular beats. Future work needs to be done to develop a noise alert algorithm to indicate the fidelity of the ECG. The downsampling blocks in the system enable each of these tasks to be operated at a much lower rate than that of the input signal. Thus the FB offers one set of filters which can be used to preprocess the ECG for various tasks.

\section{Acknowledgments}

This work was supported by a grant from Burdick Inc., Milton, WI, USA.

\section{References}

[1] Hamilton, P. S., and Tompkins, W. J., Quantitative investigation of QRS detection rules using the MIT/BIH arrhythmia database, IEEE Trans. Biomed. Eng., 1986;33:1157-1165.

[2] Barro, S., Ruiz, R., Cabello, D., and Mira, J., Algorithmic sequential decision-making in the frequency domain for life threatening ventricular arrhythmias and imitative artefacts: a diagnostic system, J. Biomed. Eng., 1989;11:320-328。

[3] Afonso, V. X., Tompkins, W. J., Nguyen, T. Q., Trautmann, S., and Luo, S., Filter bank-based processing of the stress ECG, Proc. Annu. Int. conf. IEEE Eng. Med. Biol. Soc., Sep. 1995.

[4] Afonso, V. X., Tompkins, W. J., Nguyen, T. Q., Michler, K. and Luo, S., Comparing stress ECG enhancement algorithms: with an introduction to a Filter Bank based approach, IEEE Eng. in Med. and Biol. Mag., vol. 15, no. 3, pp. $37-44,1996$.

[5] Vaidyanathan, P. P., Multirate systems and filter banks. Englewood Cliffs, NJ. Prentice-Hall, 1993.

[6] Strang, G. and Nguyen, T. Q., Wavelets and Filter Banks. Wellesley, MA, Wellesley-Cambridge Press, 1996.

[7] Soman, A. K., Vaidyanathan, P. P., and Nguyen, T. Q., Linear phase paraunitary filter banks: Theory, factorizations and designs. IEEE Trans. on Signal Processing, 1993:41:3480-3495.

[8] Malvar, H. S., Signal processing with lapped transforms, Artech House, Norwood, MA, 1992.

[9] Afonso, V. X., Tompkins, W. J., Nguyen, S., and Luo, S. Filter bank-based ECG beat detection, Proc. Annu. Int. conf. IEEE Eng. Med. Biol. Soc., Nov. 1996.

Address for correspondence:

Dr. Willis J. Tompkins

Dept. of Electrical and Computer Engineering

University of Wisconsin - Madison

1415 Engineering Drive

Madison, WI. 53706, USA

E-mail: tompkins@engr.wisc.edu 